\title{
Chemiluminescent Immunoassay versus Enzyme Linked Immunosorbent Assays for IgA Anti-Tissue Transglutaminase Antibodies Assessment in Celiac Disease Children
}

\author{
OANA BELEI ${ }^{1}$, EMIL RADU IACOB ${ }^{2 *}$, DANIELA IACOB ${ }^{3 *}$, ELENA AMARICAI ${ }^{4}$, OTILIA MARGINEAN ${ }^{1}$ \\ ${ }^{1}$ University of Medicine and Pharmacy Victor Babes Timisoara, First Pediatric Clinic, 2 Eftimie Murgu Sq., 300041, \\ Timisoara, Romania \\ ${ }^{2}$ University of Medicine and Pharmacy Victor Babes Timisoara, Pediatric Surgery Department, 2 Eftimie Murgu Sq., 300041 , \\ Timisoara, Romania \\ ${ }^{3}$ University of Medicine and Pharmacy Victor Babes Timisoara, Neonatology Department, 2 Eftimie Murgu Sq., 300041, \\ Timisoara, Romania \\ ${ }^{4}$ University of Medicine and Pharmacy Victor Babes Timisoara, Department of Rehabilitation, Physical Medicine and \\ Rheumatology, 2 Eftimie Murgu Sq., 300041, Timisoara, Romania
}

\begin{abstract}
Recent describing of atypical, silent and latent form of celiac disease (CD) increased the preocupation for screening methods.To perform a comparative study of immunoglobulin A (IgA) anti tissue-transglutaminase $(t T G)$ antibodies $(A b)$ assessment using chemiluminescence versus Enzyme Linked Immunosorbent Assay (ELISA).The study included two lots.The first lot consisted in 35 biopsy confirmed CD children aged between two and 18 years.The control lot included 40 children with normal duodenal morphology on intestinal biopsy that underwent upper digestive endoscopy for different gastrointestinal symptoms. Serum samples were provided from all subjects for IgA measurement, IgA anti tTG and EMA assessment. Immulite 2500 Anti tTG IgA (Siemens Co, LA, USA) and ImmuLisa anti-hu tTG antibody IgA ELISA (Immco Diagnostics Inc, NY, USA) kits were used for $t T G-A b$ assessment. All children underwent HLA typing for DQ2/DQ8. The sensitivity for IgA tTG assessment was greater for chemiluminescence (93,3\%) versus ELISA (86,6\%), p<0,05, while specificity, positive and negative predictive value didn't register significant differences. Statistical comparison of the two tested methods revealed a better sensitivity for chemiluminescence. Diagnosis algorithm optimisation may be obtained by associating IgA anti $t T G$-Ab assessment using chemiluminescence followed by EMA confirmation by indirect immunofluorescence for $C D$ screening.
\end{abstract}

Keywords: celiac disease, children, tisue-transglutaminase, chemiluminescence, ELISA

Celiac disease (CD) is defined as an immune-mediated enteropathy caused by intolerance to gluten in genetically susceptible individuals (associating human leukocyte antigen-HLA DQ2 or DQ8). This disease occurs in subjects presenting gastrointestinal and extradigestive symptoms. Also it can occurs in some asymptomatic subjects affected by autoimmune or genetic diseases (insulin dependent diabetes mellitus, autoimmune thyroid disorders, Down syndrome etc), patients with selective immunoglobulin A (IgA) deficiency and first-degree relatives of patients with CD. Recent researches demonstrated that the classical definition of the disease is limited to $30 \%$ of patients with genetic susceptibility and morphological changes and does not include the vast majority of subjects with gluten sensitivity, which have minor villous injuries. Most celiac cases present as atypical, silent or latent form of disease, without clinical manifestations [1].

Studies regarding atypical or silent forms of CD have generated a great interest for different methods of serological screening in gluten enteropathy. Using different serologic tests permitted a better selection of cases for intestinal biopsy in celiac patients. In clinical practice, serological tests for CD are useful in identifying patients who require intestinal biopsy findings to diagnose this condition [2]. Serological screening for CD in a population with associated risk factors is now considered the most efficient strategy, taking into account cost-effectiveness. The efficiency of this serological screening is to identify the cases with atypical or oligo-symptomatic forms of CD [2].

Some old serologic markers as IgA and IgG anti-gliadin (AGA) and anti-reticulin antibodies (Ab) have been used in the diagnosis process of $\mathrm{CD}$. These tests demonstrated suboptimal sensitivities and specificities and were excluded from routine diagnostic use [3].

\footnotetext{
*email:radueiacob@umft.ro,radueiacob@yahoo.com
} 
In 1997 a 82-85 kDa enzyme named tissue transglutaminase (tTG), was described by Dieterich et al [4] as the principal autoantigen target of IgA endomysial Ab (EMA). Consecutively, there were developed several enzyme-linked immunosorbent assay (ELISA) methods to measure IgA and IgG anti-tTG Ab [5]. An important advantage of ELISA technique is the potential for automation and the objectivity in interpretation, being a quantitative method. There are some disadvantages as nonspecific binding of antibodies to ELISA plates, resulting in a loss of specificity [5]. The use of recombinant human tTG (h-tTG), as opposed to guinea pig liver tTG (gpl-tTG) in ELISA assays has been reported to produce superior results [6]. An alternative to ELISA assay for anti-tTG Ab measurement is chemiluminescent immunoassay.

EMA and tTG antibodies assessment are both highly sensitive and highly specific tests, with values for both parameters exceeding 96\% in most studies [1] Recently, anti-deamidated gliadin peptides (DGP) assessment have replaced classic anti-gliadin tests, demonstrating a higher accuracy [2].

In 2019, The European Society for Pediatric Gastroenterology, Hepatology and Nutrition (ESPGHAN) has revised the diagnostic criteria for gluten enteropathy, underlying the importance of serological testing for this condition. According to ESPGHAN's new criteria, anti- tTG Ab serum assessment should be performed as the initial diagnostic workup in symptomatic patients when the suspicion of CD exists [7]. The standard diagnosis tool for gluten enteropathy is still intestinal biopsy showing characteristic villous lesions with few exceptions. According to ESPGHAN's revised criteria, the duodenal biopsy can be omitted in selected circumstances, based on a combination of symptoms and Ab, including symptomatic patients with IgA anti-tTG levels above $10 \mathrm{x}$ the manufacturer's cut-off, EMA positive [7].

The specificity of EMA for the diagnosis of CD was almost $100 \%$ in recent studies $[8,9]$ so this test is considered the reference standard for celiac Ab detection. EMA are detected on the smooth muscle of monkey esophagus or human umbilical tissue using indirect immunofluorescence. This assay needs microscopy and may be subject to inter-observer variability. It is known that indirect immunofluorescence technique is operator dependent and there are different sources of error: number of function hours of fluorescence source, lens quality, microscope diaphragm opening etc [8,9]. Despite these limitations, the specificity of EMA determination is very high, varying between $98 \%$ and $100 \%$ in specialized laboratories [10].

Alternative serological methods with similar or higher accuracy should be evaluated in symptomatic and atypical patients with CD and in controls in order to be validated and included in new diagnostic algorithms.

The objective of this research was to perform a comparative study of IgA anti-hu tTG Ab assessment using chemiluminescence versus ELISA assay in order to identify the more reliable test to optimize the diagnostic algorithm for CD children.

\section{Experimental part}

Material and methods

Between Jan 2015 and Aug 2019 the authors conducted a prospective study at an academic referral pediatric center from the Western part of Romania. The study included two lots. The first lot consisted in 35 biopsy confirmed CD

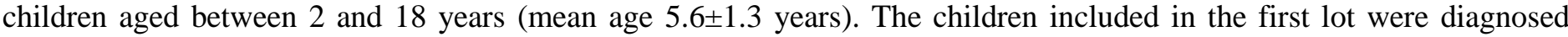
based on a screening strategy in a pediatric population with associated risk factors for CD. The second lot was the control lot and included 40 non CD children matched for age and gender, with normal duodenal morphology on intestinal biopsy that underwent upper digestive endoscopy for different gastrointestinal symptoms.

A serological screening for CD was performed among all consecutive children admitted to our hospital with symptoms or several risk factors for $\mathrm{CD}$ such as: chronic diarrhea, malabsorption syndrome, short stature, failure to thrive, iron deficiency anemia non-responsive to oral iron therapy, association of autoimmune disorders, idiopathic hypertransaminasemia, chromosomial disorders (Down syndrome, Williams syndrome, Turner syndrome), or presence of first degree relatives with $\mathrm{CD}$ in the family.

Serum samples were provided from all children submitted to screening for total IgA measurement, IgA anti-tTG and EMA Ab assessment. IgA anti-tTG Ab were tested synchronously by two techniques: ELISA, that has been routinely used in our hospital and a new chemiluminescent immunoassay. For ELISA assay, the authors used ImmuLisa ${ }^{\mathrm{TM}}$ anti-hu tTG antibody IgA ELISA kit (Immco Diagnostics Inc, NY, USA). For the chemiluminescent immunoassay, Immulite 2500 Anti tTG IgA kit was used on Immulite 2500 Analyzer (Siemens Co, LA, USA). The authors used recombinant human tTG with better accuracy, instead of murine substrate based kits in ELISA assay. In parallel, IgA EMA were tested using indirect immunoflorescence on monkeys' esophagus smooth muscle with ImmuGlo ${ }^{\mathrm{TM}}$ Anti-Endomysial Antibody (EMA) test kit (Immco Diagnostics Inc, NY, USA).

The principle of the new chemiluminescent assay used in this study on Immulite 2500 Analyzer is described below.

Immulite 2500 Anti-tTG IgA is a solid-phase, sequential chemiluminescent immunoassay. The solid phase, a 
polystyrene bead, is coated with a tTG antigen. The patient specimen and coated bead are added to the reaction tube. An alkaline phosphatase-labeled monoclonal anti-lgA antibody is also added to the reaction tube. After the wash and incubation steps, chemiluminescent substrate undergoes hydrolysis in the presence of alkaline phosphatase. Immulite 2500 Anti-tTG IgA is an immunometric assay. The photon output, as measured by the luminometer, is related to the presence of anti-tTG IgA Ab in the sample [11].

For ELISA assay, the results were interpreted using the manufacturers' recommended cut off values: $<20 \mathrm{EU} / \mathrm{mL}-$ negative; 20 - $25 \mathrm{EU} / \mathrm{mL}$ : borderline; > $25 \mathrm{EU} / \mathrm{mL}$ - positive.

For the chemiluminescent immunoassay, the results were also based on the manufacturers' recommended cut off values: : <4.0 U.mL - negative, $\geq 4.0 \mathrm{U} / \mathrm{mL}$ - positive.

For CD confirmation, all children with at least one positive serologic test underwent upper gastrointestinal endoscopy with intestinal biopsy. For each patient, two biopsy samples were obtained from the duodenal bulb and four biopsy samples from second part of the duodenum. Histological interpretation of the intestinal samples was performed by an experienced pathologist using Marsh classification modified by Oberhuber [12,13].

The control lot included 40 consecutive children matched for age and gender: 39 subjects that underwent upper digestive endoscopy for different gastrointestinal symptoms such as dysphagia, heartburn, reccurent vomiting, severe epygastralgia, portal hypertension due to portal cavernoma, upper digestive bleeding without hypovolemic shock and one child submitted to serologic screening for CD which had normal villous histology on biopsy sample. Intestinal biopsy was performed during endoscopy in all cases using the same sampling model as applied in the first lot. CD was excluded in all cases from the control lot by normal duodenal histology. All subjects from the control lot underwent the same serological tests for CD as the children included in the first lot.

Additionally, all children enrolled in both study lots underwent HLA typing for the detection of DQ2/DQ8 alleles. The authors used QIAamp DNA Blood Mini kit (Qiagen N.V.) for the extraction and isolation of the genomic deoxyribonucleic acid (DNA). An optimal concentration of genomic DNA (50 ng/ $\mu \mathrm{L}$ ) was required, in order to obtain an efficient polimerase chain reaction (PCR) amplification. The isolated genomic was analyzed using PCR-SSP (polimerase chain reaction sequence specific primers) method for the quantification of HLA DQ2 and DQ8 haplotypes. AllSet Gold HLA DQA1 (32 mix), AllSet Gold HLA DQB1 02/04 and AllSet Gold HLA DQB1 03, Dynal -Invitrogen (Life Technologies Ltd, Scotland, UK ) were used for this purpose. The interpretation of the results was done according to the specific Worksheet - Gel Documentation Form associated to the primers kit used which contained all the possible alleles combinations.

The exclusion criteria were: patients with selective IgA deficiency, subjects who were following a gluten free diet at the moment of enrollment, children with conditions that may cause non-celiac enteropathy and villous atrophy such as: parasitic infection (Giardiasis), cow's milk protein allergy, immune-mediated enteropathy, eosinophilic gastroenteritis, Human Immunodeficiency Virus (HIV) associated enteropathy and combined immunodeficiency states.

All legal guardians/parents of patients enrolled in this study have signed an informed consent prior to inclusion. The study was approved by Ethical Committee of the host institution. The work was conducted in compliance with Local Institutional Review Board for Human Subjects Research Committee requirements.

The statistical analyze was performed using a specific application - $\mathrm{R}$ statistic soft program version 2.7.1. Means and standard deviations (SD) were calculated for numerical variables with a normal distribution, whereas for variables with a non-normal distribution, medians and ranges were calculated. Data were analyzed by chi-square test. For all statistical analyses, a two-tailed $\mathrm{p}$ value $<0.05$ was considered significant. Sensitivities and specificities were determined using the manufacturer's recommended cut off points and receiver operating characteristic (ROC) analysis was performed for both test kits used for ELISA and chemiluminescent assay. The area under the curve (AUC) for each ROC plot was also calculated and compared between methods.

\section{Results and discussions}

Between Jan 2015 and Aug 2019, 875 consecutive children with associated risk factors for CD underwent serologic screening. From them, 36 children had at least one positive serologic test. The authors performed upper digestive endoscopy and intestinal biopsy to all these 36 children as described in Material and Methods section. The intestinal biopsies showed characteristic villous injuries in 35 patients, with lesions scores ranging from Marsh II to Marsh IIIc, according to Marsh classification modified by Oberhuber [12,13]. One child with moderate elevation for IgA tTG Ab on ELISA assay $(33.7 \mathrm{EU} / \mathrm{ml})$, negative IgA tTG Ab on chemiluminescent immunoassay $(3 \mathrm{U} / \mathrm{mL})$ and negative result for EMA had normal duodenal histology. The remaining 35 children had positive EMA tests and different combinations of positive/negative IgA tTG Ab tested with ELISA and chemiluminescent assays.

All 36 children with at least one positive serologic test for CD underwent HLA typing for the detection of DQ2/DQ8 
alleles. All 35 patients with suggestive histological villous alteration for gluten enteropathy associated predisposing genetic background for CD (heterozygous/ homozygous DQ2 or DQ8 haplotype). The child with normal villous aspect on biopsy sample and positive IgA tTG Ab on ELISA assay associated other HLA alleles, non DQ2/DQ8. Taking into consideration the high negative predictive value of haplotyping [7], CD was excluded in this case. The child was included in the control lot. The IgA tTG result was considered false positive when using ImmuLisa anti-hu tTG antibody IgA ELISA kit.

All 35 children with predisposing genetic background for CD and intestinal injuries classified Marsh II-IIIc were framed as active celiac patients and included in the first lot of study.

Besides the case with false positive IgA tTG on ELISA assay, the control lot included 39 children with normal duodenal histology and negative celiac serology for all biomarkers tested: negative IgA tTG Ab assessed by ELISA and chemiluminescent immunoassay and negative EMA results using indirect imunofluorescence with ImmuGlo ${ }^{\mathrm{TM}}$ AntiEndomysial Antibody (EMA) test kit. All 40 children included in the control lot were tested for HLA DQ2 and DQ8 haplotypes. All subjects associated different HLA alleles combinations, non DQ2 /DQ8, strengthening the exclusion of gluten enteropathy in all these cases. Negative results for all antibodies on gluten containing diet or absence of both HLA DQ2 and HLA DQ8 certainly exclude the presence of CD.

The conclusions of upper digestive endoscopy for those 39 children included in the control lot were: nine cases with antral or pangastritis (Helicobacter pylori positive or negative), five cases with gastric and/or duodenal ulcers, nine cases with Mallory-Weiss syndrome, three cases with second degree esophageal varices withour bleeding, three cases with erosive esophagitis in context of gastroesophageal reflux disease, two cases with Schatzki rings passably with pediatric endoscope and eight cases with normal mucosal appearance from the esophagus to the second part of the duodenum.

Due to ethical reasons, healthy children cannot be referred to undergo upper digestive endoscopy in order to prove the absence of CD based on normal intestinal morphology. So the control lot in this study included consecutive children with gastro-intestinal symptoms that have been submitted to upper digestive endoscopy for well established reasons, unrelated to celiac disease.

Assessing the serological results, the authors calculated the sensitivity (Sn), specificity (Sp), positive predicted value (PPV) and negative predicted value (NPV) for: ImmuLisa ${ }^{\mathrm{TM}}$ anti-hu tTG antibody IgA ELISA kit, Immulite 2500 Anti tTG IgA kit and ImmuGlo ${ }^{\text {TM }}$ Anti-Endomysial Antibody test kit.

The accuracy of different methods used for IgA anti tTG Ab and EMA measurement in CD children and controls included in this study was based on histological findings and HLA typing.

Table 1 depicts the statistical parameters for all three test kits used for serological biomarkers assessment in this study.

Table 1

THE STATISTICAL PARAMETERS FOR ALL THREE TEST KITS USED FOR SEROLOGICAL BIOMARKERS ASSESSMENT IN THIS STUDY

\begin{tabular}{|c|c|c|c|c|}
\hline Kit & $\mathrm{Sn}$ & $\mathrm{Sp}$ & $\mathrm{PPV}$ & NVP \\
\hline $\begin{array}{c}\text { ImmuGlo Anti-Endomysial } \\
\text { Antibody (EMA) Test }\end{array}$ & $100 \%$ & $100 \%$ & $100 \%$ & $100 \%$ \\
\hline $\begin{array}{c}\text { ImmuLisa anti-hu tTG antibody } \\
\text { IgA ELISA }\end{array}$ & $86.6 \%$ & $99.3 \%$ & $92.8 \%$ & $\mathbf{9 8 . 7 \%}$ \\
\hline $\begin{array}{c}\text { Immulite 2500 Anti tTG IgA } \\
\text { (chemiluminescent immunoassay) }\end{array}$ & $93.3 \%$ & $99.3 \%$ & $93.3 \%$ & $\mathbf{9 9 . 3 \%}$ \\
\hline
\end{tabular}

ELISA: Enzyme Linked Immunosorbent Assay, EMA: endomysial antibody, NPV: negative predicted value, PPV: positive predicted value, Sn: sensitivity, Sp: specificity,tTG: tissue transglutaminase

EMA had $100 \%$ Sn, Sp, PPV and NPV. Our results confirmed that EMA has the highest reliability for the diagnosis of CD.

The test kit used for chemiluminescent immunoassay had a significant higher $\mathrm{Sn}$ than the test kit used for ELISA assay for IgA anti tTG Ab measurement. There weren't significant differences between Sp, PPV and NPV of both methods used for IgA anti-tTG Ab detection ( $\mathrm{p}>0.05)$.

In order to compare the $\mathrm{Sn}$ of both $\mathrm{IgA}$ anti tTG Ab test methods, statistical data processing was made by using $\mathrm{R}$ statistic soft program version 2.7.1. We realized the receiver operating characteristic (ROC) curves for ELISA method and chemiluminescence assay respectively and we calculated the area under the curve (AUC).

Figure 1 represents the ROC curve for ImmuLisa ${ }^{\text {TM }}$ anti-hu tTG IgA ELISA kit. 


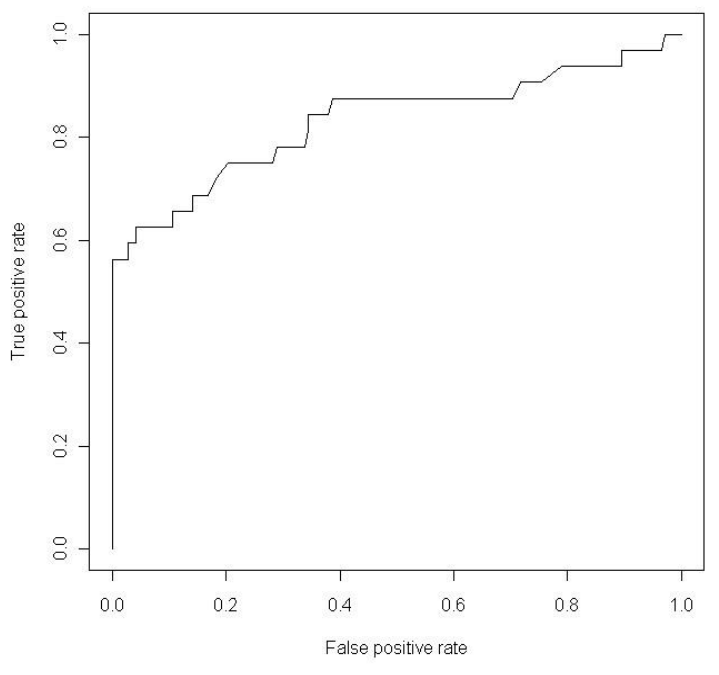

Fig. 1. The ROC curve for ImmuLisa ${ }^{\mathrm{TM}}$ anti-hu tTG IgA ELISA kit

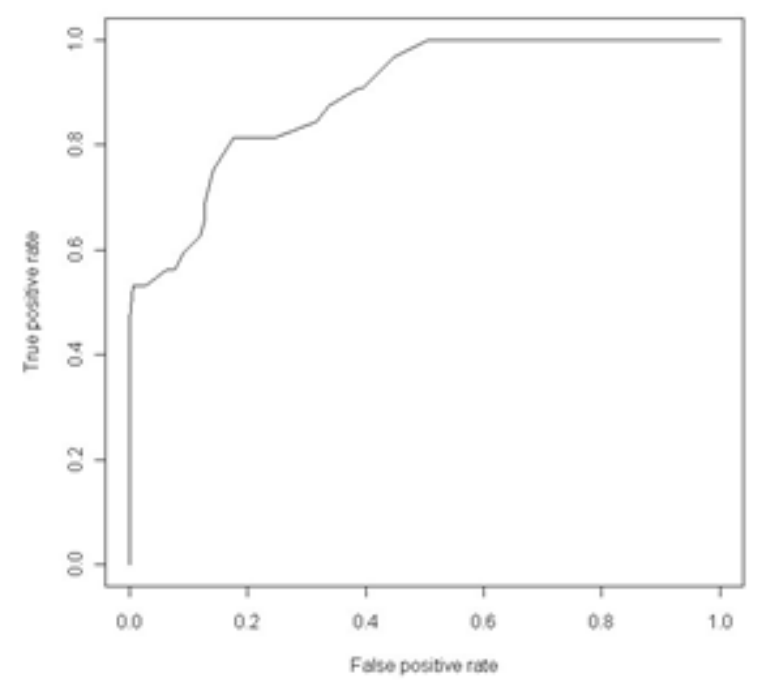

Fig.2. represents the ROC curve for Immulite 2500 Anti tTG IgA kit

For ImmuLisa $^{\mathrm{TM}}$ anti-hu tTG antibody IgA ELISA kit, AUC was 0.8316461 and for Immulite 2500 Anti tTG IgA kit AUC was 0.8945863 , indicating a statistically significant higher $\mathrm{Sn}$ for chemiluminescence assay compared to ELISA for measuring IgA anti tTG Ab.

It has been postulated that measuring IgA tTG or EMA is less accurate than IgA or IgG AGA to detect CD in children younger than two years old, leading therefore to false negative results [9]. Conventional gliadin antibody tests have, in general, low specificity and sensitivity. Some evidence exists, however, that AGA and DGP sensitivity may be higher in children younger than two years old in comparison with EMA and anti tTG Ab [14]. Due to these evidences indicating in some circumstances lower accuracy for IgA anti tTG Ab assessment, the authors included in this study children older than two years old. So, the comparisson between the two methods for IgA tTG Ab assessment - ELISA and chemiluminescence was free of bias.

Testing for serum IgA anti tTG Ab is the recommended initial approach to diagnose gluten enteropathy. Several studies confirmed that high serum concentrations of anti tTG Ab may predict villous atrophy better than low values $[15,16]$.

The clinical significance of positive anti tTG Ab test should be confirmed by intestinal biopsy and duodenal histology assessment, unless specific conditions are fulfilled to omit the biopsy [7,17]. Also, anti- tTG Ab positive results should be verified by EMA. If histology reveals lesions that are compatible with $\mathrm{CD}$, then the diagnosis will be confirmed [7].

Low levels of anti-tTG Ab have been described in several disorders unrelated to $\mathrm{CD}$, such as other autoimmune diseases, infections, tumors, myocardial damage, liver disorders, and psoriasis $[18,19]$.

According to ESPGHAN, in order to avoid unnecessary biopsies in subjects with low CD specific antibody levels $(<3$ times upper limit of normal -ULN) it is recommended that more specific tests as EMA to be performed. If EMA result is positive, intestinal biopsy should be performed. If EMA test is negative, serological tests should be repeated on a normal gluten-containing diet after three to six months [7]

In this study, the child submitted to serologic screening with normal duodenal histology included further in the control lot, had mild elevation of IgA anti tTG Ab using ELISA $(<3$ times ULN) and the result was negative when using the chemiluminescent assay. Also, EMA was negative and he associated nonDQ2/DQ8 alleles. CD was easily excluded in this case and IgA anti- tTG Ab on ELISA assay was considered false positive. Even though EMA was negative, the authors performed in this case intestinal biopsy and HLA typing to strengthen the exclusion of CD and to remove any error factors from further analyze regarding the accuracy of each tested method.

A lot of papers assessed the accuracy of different serum biomarkers for CD diagnosis and the majority compared $\operatorname{IgA}$ and/or IgG tTG and DGP with EMA. In the hierarchy of antibodies, EMA had the highest accuracy [5,6,9,10]. 
There are only a few studies in the recent literature comparing the performance of standardized commercial ELISA and chemiluminescence immunoassays for measuring IgA anti-tTG Ab serum level. Aita et al [20] compared the performance of a novel chemiluminescent tool in the detection of tTG and DGP (Q-Flash, Inova) with that of the established ELISA (Q-Lite, Inova) method. The authors found that IgA tTG measurement by chemiluminescent assay was the most accurate method. The Sn was $96.1 \%$ and $\mathrm{Sp}$ was $97 \%$ for diagnosing $\mathrm{CD}$, and it was the only variable to be significantly correlated with CD at binary logistic regression analysis ( $\mathrm{r}=0.263, \mathrm{p}<0.0001)$. Also, Q-Flash $\operatorname{IgA}$ tTG and DGP screen were more accurate than Q-Lite IgA tTG in monitoring CD patients on GFD ( $\mathrm{p}=0.003)$ [20].

Daves et al [21] evaluated a new chemiluminescence assay for measuring anti-transglutaminase (tTG) and antiendomysium (EMA) antibodies in a general population of unselected outpatients. IgA and IgG anti-tTG Ab (Quanta Flash ${ }^{\circledR} \operatorname{IgA}$ and Quanta Flash ${ }^{\circledR}$ IgG tTG, Inova Diagnostics, San Diego, CA, USA) were measured with the fullyautomated BIO-FLASH analyzer (Inova Diagnostics) in serum samples of 727 consecutive patients without a diagnosis of CD. Data were compared with those of EMA obtained in the same population. The concordance (99\%) and inter-rater agreement (Kappa Statistics, 0.943; p <0.001) between anti-tTG IgA and EmA antibodies were excellent, with sensitivity and specificity of $99 \%$ and $100 \%$, respectively. The authors concluded that Quanta Flash IgA tTG assay alone may be regarded as a reliable approach for screening of $\mathrm{CD}$, with no need to perform EMA detection [21].

Habtamu et al [22] designed a novel immunosensor with application for CD diagnosis, based on an electrogenerated chemiluminescence readout, using membrane-templated gold nanoelectrode ensembles as a detection platform. The sensor was applied to the analysis of anti-tTG in human serum samples, showing to be suitable to discriminate between healthy and celiac patients [22].

According to other recent publications, our results indicated a better performance for chemiluminescent immunoassay (Immulite 2500 Anti tTG IgA kit) compared to standard ELISA (ImmuLisa anti-hu tTG antibody IgA kit) for measuring IgA tTG serum levels.

The classic form of celiac disease associating chronic diarrhea in infants is still a common condition for addressability to pediatric gastroenterologists [23], therefore it is important to have reliable laboratory methods with high accuracy for serologic screening.

If anti tTG Ab serum levels are moderately elevated - less than three times ULN, a false-positive result must be taken into consideration. In these subjects, EMA assessment may help differentiating between false positive and true-positive levels. Based on the results of this study and the conclusions of other publications, we recommend to replace standard ELISA method with chemiluminescence immunoassay for measuring IgA tTG Ab serum level. In the algorithm of CD screening in symptomatic or asymptomatic children, positive IgA tTG results should always be confirmed by EMA testing.

\section{Conclusions}

Statistical comparison of the two tested methods revealed a better sensitivity for chemiluminescence. Diagnosis algorithm optimisation may be obtained by associating IgA anti tTG-Ab assessment using chemiluminescence for CD screening followed by EMA confirmation by indirect immunofluorescence. HLA typing can be useful to exclude the possibility of $\mathrm{CD}$ development in patients at high risk and can provide additional information if the clinical or serological picture is unclear.

\section{References}

1.JERICHO, H., ASSIRI, A., GUANDALINI, S., Celiac Disease and Wheat Intolerance Syndrome: A Critical Update and Reappraisal., J. Pediatr. Gastroenterol. Nutr., 64(1), 2017, p.15-21.

2.BELEI, O., MARGINEAN, O., The Usefulness of IgA/IgG DGP/tTG Screen Assay for Celiac Disease Detection among Symptomatic and at Risk Young Children., International Journal of Celiac Disease, DOI: 10.12691/ijcd-1-1-9, 1(1), 2013, p.23-26.

3.NANDIWADA, S.L., TEBO, A.E., Testing for antireticulin antibodies in patients with celiac disease is obsolete: a review of recommendations for serologic screening and the literature., Clin. Vaccine Immunol., 20, 2013, p.447-451.

4.DIETERICH, W., EHNIS, T., BAUER, M., DONNER, P., VOLTA, U., RIECKEN, E.O., SCHUPPAN, D., Identification of tissue transglutaminase as the autoantigen of celiac disease., Nat. Med., 3,1997, p.797-801.

5.FEIGHERY, C., CONLON, N., JACKSON, J., Adult population screening for coeliac disease: comparison of tissue-transglutaminase antibody and anti-endomysial antibody tests., European Journal of Gastroenterology \& Hepatology, Vol. 18, No. 11, 2006, p.1173-1175.

6.WONG, C.W., WILSON, R.J., STEELE, R.H., RADFORD-SMITH, G., ADELSTEIN, S., A comparison of 13 guinea pig and human anti-tissue transglutaminase antibody ELISA kits., J. Clin. Pathol., 55, 2002, p.488-494.

7.HUSBY, S., et al., European Society Paediatric Gastroenterology, Hepatology and Nutrition guidelines for diagnosing coeliac disease 2019., J. Ped. Gastroent. Nutr., 2019, Publish Ahead of Print

8.BRUSCA, I., Overview of biomarkers for diagnosis and monitoring of celiac disease., Adv. Clin. Chem., doi: 10.1016/bs.acc.2014.12.006, 68, 2015, p.1-55. 
9.GIERSIEPEN, K., LELGEMANN, M., STUHLDREHER, N., RONFANI, L., HUSBY, S., KOLETZKO, S., KORPONAY-SZABÓ, I.R., ESPGHAN Working Group on Coeliac Disease Diagnosis, Accuracy of diagnostic antibody tests for coeliac disease in children: summary of an evidence report., J. Pediatr. Gastroenterol. Nutr., doi: 10.1097/MPG.0b013e318216f2e5, Feb., 54(2), 2012, p.229-241.

10.LEWIS, N.R., SCOTT, B.B., Systematic review: the use of serology to exclude or diagnose coeliac disease (a comparison of the endomysial and tissue transglutaminase antibody tests)., Aliment. Pharmacol. Ther., 24, 2006, p.47-54.

11.INSERT IMMULITE 2500 Anti-tTG IgA (PIL5KTD-3, 2006-12-29), Siemens Medical Solutions Diagnostics, LA, USA

12.OBERHUBER, G., et al., The histopathology of coeliac disease: time for a standardized report scheme for pathologists., Eur. J. Gastroenterol. Hepatol., 11, 1999, p.1185-1194.

13.CORAZZA,, G.R., et al., Coeliac disease., J. Clin. Pathol., 58, 2005, p.573-574.

14.LAGERQVIST, C., DAHLBOM, I., HANSSON, T., JIDELL, E., JUTO, P., OLCEN, P., et al., Antigliadin immunoglobulin A best in finding celiac disease in children younger than 18 months of age., J. Pediatr. Gastroenterol. Nutr., 47, 2008; p.428-435.

15.SINGH, P., KURRAY, L., AGNIHOTRI, A., DAS, P., VERMA, A.K., SREENIVAS, V., DATTAGUPTA, S., MAKHARIA, G.K., Titers of anti-tissue transglutaminase antibody correlate well with severity of villous abnormalities in celiac disease., J. Clin. Gastroenterol., doi:10.1097/MCG.0000000000000105, Mar. 49(3), 2015, p.212-217.

16.RAHMATI, A., SHAKERI, R., SOHRABI, M., ALIPOUR, A., BOGHRATIAN, A., SETAREH, M., ZAMANI, F., Correlation of tissue transglutaminase antibody with duodenal histologic Marsh grading., Middle East J. Dig. Dis., Jul, 6(3), 2014, p.131-136.

17.VIVAS, S., RUIZ DE MORALES, J.G., RIESTRA, S., et al., Duodenal biopsy may be avoided when high transglutaminase antibody titers are present., World J. Gastroenterol., 15, 2009, p.4775-4780.

18.FERRARA, F., QUAGLIA, S., CAPUTO, I., et al., Anti-transglutaminase antibodies in non-coeliac children suffering from infectious diseases., Clin. Exp. Immunol., 159, 2010, p.217-223.

19.COMERFORD, R., KELLY, J., FEIGHERY, C., BYRNE, G., IgG anti-tTG responses in different autoimmune conditions differ in their epitope targets and subclass usage., Mol. Immunol., doi:10.1016/j.molimm.2015.06.026., Oct., 67(2 Pt B), 2015, p.369-376.

20.AITA, A., ROSSI, E., BASSO, D., GUARISO, G., BOZZATO, D., PELLOSO, M., PESCARIN, M., ZAMBON, C.F., NAVAGLIA, F., GRECO, E., GASPARETTO, M., FOGAR, P., PADOAN, A., MOZ, S., PLEBANI, M., Chemiluminescence and ELISA-based serum assays for diagnosing and monitoring celiac disease in children: a comparative study., Clin. Chim. Acta., doi:10.1016/j.cca.2013.03.024., Jun., 5, 421, 2013, p. 202-207.

21.DAVES, M., CEMIN, R., PERKMANN, V., BERNARD, P., CAPRIOLI, G., PLATZGUMMER, S., LIPPI, G., Fully-automated, chemiluminescence IgA and IgG anti-tissue transglutaminase (tTG) antibodies serum assays for the screening of celiac disease., J. Immunol. Methods., doi:10.1016/j.jim.2016.01.002., Feb., 429, 2016, p.57-59.

22.HABTAMU, H.B., SENTIC, M., SILVESTRINI, M., DE LEO, L., NOT, T., ARBAUlt, S., MANOJLOVIC, D., SOJIC, N., UGO, P., A Sensitive Electrochemiluminescence Immunosensor for Celiac Disease Diagnosis Based on Nanoelectrode Ensembles., Anal. Chem., doi:10.1021/acs.analchem.5b02801, Dec., 15;87(24), 2015, 12080-12087.

23.BELEI, O.A., HEREDEA, E.R., BOERIU, E., MARCOVICI, T.M., CERBU, S., MĂRGINEAN, O., IACOB, E.R., IACOB, D., MOTOC, A.G.M., BOIA, E.S., Verner-Morrison syndrome. Literature review., Rom. J. Morphol. Embryol., 58(2), 2017, p.371-376.

$\overline{\text { Manuscript received: } 28.10 .2019}$ 